\title{
SEMESTRE EUROPEO: LA SUPERVISIÓN DE LAS POLÍTICAS ECONÓMICAS, PRESUPUESTARIAS Y FISCALES DEL ESTADO ESPAÑOL
}

\author{
Covadonga FerRer Martín DE VidAles \\ Departamento de Derecho Constitucional \\ Facultad de Derecho \\ Universidad Complutense de Madrid \\ cferrerm@ucm.es
}

\begin{abstract}
RESUMEN
El presente trabajo analiza la supervisión de las politicas económicas, fiscales y presupuestarias del Estado español llevada a cabo en el marco del Semestre Europeo, con el objeto de identificar los principales ámbitos en los que ha sido necesario realizar reformas para cumplir con las orientaciones y prioridades acordadas en el mismo.

Palabras clave: Semestre Europeo, gobernanza económica, políticas económicas, fiscales y presupuestarias.
\end{abstract}

\section{ABSTRACT}

The current paper examines the supervision of economic, budgetary and fiscal policies of the Spanish State carried out within the framework of the European Semester, with the aim to identify the main areas were essential amendements have been introduced in order to comply with the guidelines and priorities issued from the EU level.

Keywords: European Semester, economic governance, economic, budgetary and fiscal policies.

\section{ZUSSAMENFASSUNG}

Die vorliegende Arbeit beschäftigt sich mit der Überprüfung der Wirtschafts-, Haushalts- und Steuerpolitik im Rabmen des Europäischen Semesters. Ibr Ziel ist es, die wesentlichen Bereiche aufzuzeigen, in welchen es nötig wurde, Erneuerungen vorzunebmen, um die Zielrichtungen und Prioritäten einzubalten, die während desselben vereinbart wurden.

Schlüsselwörter: Europäisches Semester, Governance, Wirtschaft, Wirtschaftspolitik, Steuerpolitik, Haushaltspolitik. 
SUMARIO: I. INTRODUCCIÓN.-II. LA NUEVA GOBERNANZA EUROPEA.III. EL SEMESTRE EUROPEO.-IV. LA SUPERVISIÓN DE ESPAÑA DENTRO DEL NUEVO SEMESTRE EUROPEO DE COORDINACIÓN ECONÓMICA.-1. Semestre Europeo 2011.-2. Semestre Europeo 2012.-3. Semestre Europeo 2013.- 4. Semestre Europeo 2014.-5. Semestre Europeo 2015.6. Semestre Europeo 2016. - 7. Semestre Europeo 2017. - 8. El Semestre Europeo en 2018.- - V. CONCLUSIONES.

\section{INTRODUCCIÓN}

Como es ya de sobra conocido, la crisis económica y financiera mundial que estalló en 2008 puso de relieve la ineficiencia e inadecuación de los mecanismos de una Unión Económica y Monetaria (en adelante, UEM) incompleta, en la que todavía no se habían dado los pasos hacia una unión económica, presupuestaria y fiscal ${ }^{1}$. Para tratar de solventar los mismos, los Estados miembros comenzaron a trabajar en una serie de nuevas medidas destinadas a reformar las reglas y el funcionamiento de dicha UEM, así como a reforzar la gobernanza económica de la Unión Europea ${ }^{2}$.

Las citadas medidas implican una mayor coordinación y vigilancia de las políticas económicas, fiscales y presupuestarias de los Estados miembros a través del nuevo Semestre Europeo. La supervisión y coordinación de las distintas políticas ha quedado reforzada, en particular la de la política económica de los Estados miembros.

A la cabeza de todas estas nuevas medidas se ha situado el Consejo Europeo, que ha dado orientaciones precisas y ha demandado al Consejo, al Parlamento Europeo y a la Comisión la adopción de las decisiones pertinentes, imponiéndoles incluso plazos. En su reunión de marzo de 2010, el Consejo Europeo pidió a su presidente que estableciese, en cooperación con la Comisión, un grupo especial de trabajo que presentase al Consejo las medidas necesarias para alcanzar el objetivo de un marco mejorado de resolu-

${ }^{1}$ El Tratado de la Unión Europea de 1992 consagraba una unión monetaria completa, pero no una unión económica y fiscal. La política fiscal siguió siendo competencia de los Estados miembros, si bien mediante el Pacto de Estabilidad y Crecimiento (en adelante, PEC) se establecieron estrictos límites para el control de los déficits públicos y del nivel de deuda pública de los mismo. Además, se prohibía al Banco Central Europeo (en adelante, BCE) financiar directamente los déficits de los Estados y se impuso la regla de no asistencia entre países (no bail-out). Vid. M. De LA Rocha VázQuez, El futuro de la Unión Económica y Monetaria Europea: ¿qué ha fallado y qué reformas se requieren?, documento de trabajo 54/2010, Madrid, OPEX-Fundación Alternativas, 2010, pp. 7-8.

${ }^{2}$ Ibid., p. 5. 
ción de crisis y una mejor disciplina presupuestaria ${ }^{3}$. Grupo que presentó un informe final a la cumbre de octubre de 2010, que fue refrendado por el Consejo Europeo, que indicó, asimismo, que las recomendaciones previstas por el citado informe deberían adoptarse legislativamente para el verano de 2011, siguiendo un planteamiento de vía rápida ${ }^{4}$. De esta forma, las recomendaciones del Grupo Especial sobre Gobernanza Económica dieron lugar a la adopción del paquete legislativo de seis medidas denominado six pack, paquete de medidas legislativas mediante el que se codifica el Semestre Europeo. Y fue también el Consejo Europeo quien decidió que era necesario estudiar dos nuevas propuestas de la Comisión para reforzar el mismo, conocidas como two pack ${ }^{5}$, y que estuviesen aprobadas en verano de 2012.

El objeto del presente trabajo es analizar la supervisión en el marco del citado Semestre Europeo de las políticas económicas, fiscales y presupuestarias del Estado español, con el objeto de identificar los principales ámbitos en los que España ha debido llevar a cabo reformas de cara a cumplir con las orientaciones, prioridades y recomendaciones acordadas dentro de dicho marco.

\section{LA NUEVA GOBERNANZA EUROPEA}

Como se ha adelantado, la crisis económica ha obligado a adoptar toda una serie de medidas para reformar las reglas y el funcionamiento de la UEM, y reforzar la gobernanza económica de la Unión Europea ${ }^{6}$. Dentro de las diversas medidas adoptadas encontramos, por un lado, las medidas e instrumentos para una mayor coordinación y supervisión de las políticas económicas, fiscales y presupuestarias de los Estados miembros, y, por otro, las medidas para salvaguardar la estabilidad financiera de la zona euro y las destinadas a sanear el sector financiero.

3 Vid. punto 7 de las Conclusiones del Consejo Europeo de 25 y 26 de marzo de 2010.

4 Vid. punto 1 de las Conclusiones del Consejo Europeo de 28 y 29 de octubre de 2010. En su reunión de febrero de 2011, el Consejo Europeo volvía a señalar que el acuerdo debía alcanzarse antes de finales de junio de 2011. Vid. punto 27 de las Conclusiones del Consejo Europeo de 4 de febrero de 2011.

5 Vid. puntos 1 y 2 de las Conclusiones del Consejo Europeo de 9 de diciembre de 2011.

${ }^{6}$ Algunas de ellas ya figuraban en el informe final presentado en diciembre de 2012 por el presidente Van Rompuy a la cumbre del Consejo Europeo para la evolución hacia una arquitectura más sólida de la UEM, la cual basaba en cuatro pilares: un marco financiero integrado, un marco presupuestario integrado, un marco integrado de política económica y el refuerzo de la legitimidad democrática y la rendición de cuentas. Vid. H. VAN Rompuy, Hacia una auténtica Unión Económica y Monetaria, Bruselas, 2012. 
Por lo que respecta a las primeras, que son las que interesan al objetivo del presente estudio, las nuevas normas se encuentran recogidas en los denominados six-pack ${ }^{7}$ y two-pack ${ }^{8}$, paquetes de medidas legislativas que refuerzan la supervisión de las políticas económicas, fiscales y presupuestarias de los Estados miembros (con previsiones específicas para los Estados de la zona euro), y el Tratado de Estabilidad, Coordinación y Gobernanza (en adelante, TECG), que refuerza a los dos paquetes anteriores. Las citadas normas se aplican en el contexto del Semestre Europeo, nuevo ciclo para la coordinación de la políticas económicas y presupuestarias de los Estados miembros'. A ellos se añade el Pacto por el Euro Plus, cuyo objetivo es también reforzar el pilar económico de la Unión Europea.

Mediante los mismos se establecen mecanismos que buscan, por un lado, prevenir y, por otro, corregir. Prevenir, de forma que los problemas económicos de un Estado miembro no empeoren y afecten al resto de Esta-

${ }^{7}$ El six-pack está integrado por seis medidas legislativas: los Reglamentos (UE) núms. 1175/2011 y 1177/2011, que reforman el PEC [Reglamento (UE) núm. 1175/2011 del Parlamento Europeo y del Consejo, de 16 de noviembre de 2011, por el que se modifica el Reglamento (CE) núm. 1466/97 del Consejo, relativo al refuerzo de la supervisión de las situaciones presupuestarias y a la supervisión y coordinación de las políticas económicas (DO, núm. L 306, de 23 de noviembre de 2011), y Reglamento (UE) núm. 1177/2011 del Consejo, de 8 de noviembre de 2011, por el que se modifica el Reglamento (CE) núm. 1467/97, relativo a la aceleración y clarificación del procedimiento de déficit excesivo (DO, núm. L 306, de 23 de noviembre de 2011)]; el Reglamento (UE) núm. 1176/2011, que crea el Procedimiento de Desequilibrio Macroeconómico para la detección de forma temprana de dichos desequilibrios [Reglamento (UE) núm. 1176/2011 del Parlamento Europeo y del Consejo, de 16 de noviembre de 2011, relativo a la prevención y corrección de los desequilibrios macroeconómicos (DO, núm. L 306, de 23 de noviembre de 2011)]; el Reglamento (UE) núm. 1174/2011, que establece un sistema de sanciones para la corrección efectiva de los desequilibrios macroeconómicos en la zona euro [Reglamento (UE) núm. 1174/2011 del Parlamento Europeo y del Consejo, de 16 de noviembre de 2011, relativo a las medidas de ejecución destinadas a corregir los desequilibrios macroeconómicos excesivos en la zona euro (DO, núm. L 306, de 23 de noviembre de 2011)], y la Directiva 2011/85/UE, que introduce normas detalladas sobre las características que deben tener los marcos presupuestarios de los Estados miembros para garantizar el cumplimiento de la obligación de evitar déficits públicos excesivos (Directiva 2011/85/UE del Consejo, de 8 de noviembre de 2011, sobre los requisitos aplicables a los marcos presupuestarios de los Estados miembros).

8 Reglamento (UE) núm. 473/2013 del Parlamento Europeo y del Consejo, de 21 de mayo de 2013, sobre disposiciones comunes para el seguimiento y la evaluación de los proyectos de planes presupuestarios y para la corrección del déficit excesivo de los Estados miembros de la zona del euro (DO, núm. L 140, de 27 de mayo de 2013), y Reglamento (UE) núm. 472/2013 del Parlamento Europeo y del Consejo, de 21 de mayo de 2013, sobre el reforzamiento de la supervisión económica y presupuestaria de los Estados miembros de la zona euro cuya estabilidad financiera experimenta o corre el riesgo de experimentar graves dificultades (DO, núm. L 140, de 27 de mayo de 2013).

9 Vid. European Commission, «The EU's economic governance explained», MEMO/15/6071, Brussels, 26 de noviembre de 2015. 
dos miembros de la Unión ${ }^{10}$, lo que se consigue mediante las normas introducidas por el Reglamento (UE) núm. 1175/2011, que reforma la vertiente preventiva del Pacto de Estabilidad y Crecimiento (en adelante, PEC) con el objetivo de prevenir, en una fase temprana, que se produzcan déficits públicos excesivos y fomentar la supervisión y coordinación de las políticas económicas ${ }^{11}$; mediante el Procedimiento de Desequilibrio Macroeconómico (en adelante, PDM), mecanismo de alerta para la detección de desequilibrios macroeconómicos de forma temprana que trata de prevenir los mismos o de corregir los que ya se estén produciendo ${ }^{12}$; así como por las previsiones adoptadas en el marco del TECG, mediante las que se persigue promover la disciplina presupuestaria, reforzar la coordinación de las políticas económicas y mejorar la gobernanza de la zona euro ${ }^{13}$, e incorporar al Derecho nacional el principio de equilibrio presupuestario (la llamada «regla de oro») mediante disposiciones con fuerza vinculante y carácter permanente, preferentemente de rango constitucional ${ }^{14}$. Corregir, para reforzar las nuevas normas de gobernanza económica y asegurarse de que los Estamos miembros llevan a cabo las medidas necesarias para corregir los mencionados problemas económicos ${ }^{15}$, mediante el Procedimiento de Déficit Excesivo, cuyo objetivo es disuadir los déficits públicos excesivos y propiciar su pronta corrección en caso de que se produzcan ${ }^{16}$; así como mediante la vertiente correctiva del PDM, el Procedimiento de Desequilibrio Excesivo (en adelante, PDE), que permite al Consejo adoptar una recomendación estableciendo la existencia de un desequilibrio excesivo y recomendando al Estado que adopte medidas correctoras ${ }^{17}$.

Todas estas medidas enmarcadas en la nueva gobernanza económica europea se aplican a lo largo de un ciclo anual dividido en dos par-

10 Vid. European Commission, «How the EU monitors national economic policies», disponible en www.ec.europa.eu (consultado el 22 de mayo de 2018).

${ }_{11}$ Art. 1 del Reglamento (CE) núm. 1466/97, tal y como ha sido modificado por el Reglamento (UE) núm. 1175/2011. La versión consolidada puede consultarse en ELI, bttp://data.europa.eu/eli/reg/1997/1466/2011-12-13.

12 Vid. art. 3 del Reglamento (UE) núm. 1176/2011 del Parlamento Europeo y del Consejo, de 16 de noviembre de 2011, relativo a la prevención y corrección de los desequilibrios macroeconómicos (DO, núm. L 306, de 23 de noviembre de 2011).

13 Vid. art. 1 del Tratado de Estabilidad, Coordinación y Gobernanza en la Unión Económica y Monetaria, de 2 de marzo de 2012, también llamado «Pacto Presupuestario».

${ }_{14} \mathrm{O}$ garantizar su respecto y cumplimiento plenamente de otro modo a lo largo de los procedimientos presupuestarios nacionales. Vid. ibid., art. 3.2.

${ }^{15} \mathrm{Vid}$. European Commission, «How the EU monitors national...», op. cit.

16 Art. 1 de la versión consolidada del Reglamento (CE) núm. 1467/2011.

17 Vid. art. 7 del Reglamento (UE) núm. 1176/2011. 
tes: el Semestre Europeo y el Semestre Nacional ${ }^{18}$. Por tanto, pasamos a continuación a examinar el Semestre Europeo, una de las medidas, como hemos señalado, que se ha adoptado para incrementar el control y supervisión de las políticas económicas, presupuestarias y fiscales de los Estados miembros.

\section{EL SEMESTRE EUROPEO}

El Semestre Europeo es el nuevo ciclo para la coordinación de la políticas económicas y presupuestarias de los Estados miembros, cuyo objetivo es asegurar que los mismos discuten sus planes económicos y presupuestarios a lo largo del año, y que los ajustan a los objetivos y normas acordados a nivel de la Unión ${ }^{19}$.

Como se ha adelantado, es fruto de la labor de un grupo especial de trabajo creado por mandato del Consejo Europeo en su cumbre de marzo de $2010^{20}$ y ha sido codificado a través del six-pack, paquete adoptado también como resultado de los trabajos del citado grupo especial, que ha sido reforzado mediante el two-pack. A su vez, ambos son reforzados por el TECG.

El ciclo previsto por el Semestre Europeo comienza a principios de año, cuando se preparan las orientaciones políticas generales a escala de la Unión que habrán de seguir todos los Estados miembros, si bien previamente se lleva a cabo una fase preparatoria (entre noviembre y diciembre) en la que se evalúa la situación y progresos del año anterior y en la que se publican el Estudio Prospectivo Anual sobre el Crecimiento de la Comisión (en adelante, EPAC), en el que se presentan las prioridades de política económica claves para el año siguiente; el Informe sobre el Mecanismo de Alerta (en adelante, IMA), para evitar riesgos potenciales y prevenir la aparición de desequilibrios macroeconómicos que puedan ser perjudiciales, así como la corrección de los existentes, y el Informe conjunto sobre el empleo (en adelante, ICE), que analiza la situación del mismo y las respuestas adoptadas por los Estados miembros ${ }^{21}$.

${ }^{18}$ European Commission, «How the EU monitors national...», op. cit.

19 Vid. Comisión Europea, «La gobernanza económica de la UE al detalle», MEMO, Bruselas, 2014. Un esquema de la línea temporal del Semestre Europeo se encuentra disponible en la web de la Comisión: bttps://ec.europa.eu/info/sites/info/ files/2018-european-semester-timeline_en.pdf.

${ }^{20}$ Vid. punto 7 de las Conclusiones del Consejo Europeo de 25 y 26 de marzo de 2010.

21 Vid. Consejo Europeo/Consejo de la Unión Europea, «Cómo funciona el Semestre Europeo», disponible en www.consilium.europa.eu (consultado el 27 de enero de 2016). 
Además, teniendo en cuenta el IMA, la Comisión puede realizar un examen exhaustivo de los Estados miembros en los que el riesgo de desequilibrios macroeconómicos sea elevado. Asimismo, en esta fase preparatoria se presenta el Proyecto de recomendación para la zona euro, en el que se invita a los Estados miembros de la eurozona a aplicar políticas específicas ${ }^{22}$.

A continuación, durante los meses de enero y febrero, el Consejo de la Unión Europea, que adopta directrices y conclusiones, y el Parlamento Europeo debaten el EPAC de la Comisión. Asimismo, se aprueba el proyecto de recomendación para la zona euro ${ }^{23}$.

En marzo, el Consejo Europeo formula las orientaciones en materia de política económica y presupuestaria que han de seguir los Estados miembros. Se trata de las orientaciones que el Consejo Europeo adopta en la denominada cumbre de primavera y sobre las cuales el Consejo de Asuntos Económicos y Financieros de la Unión Europea (en adelante, ECOFIN) abre el procedimiento formal establecido en el art. 121.2 del Tratado de Funcionamiento de la Unión Europea (en adelante, TFUE) y formula un proyecto de orientaciones generales sobre el que el Consejo Europeo discute una conclusión en su reunión de junio ${ }^{24}$.

Asimismo, la Comisión publica en marzo los informes por país, en los que analiza la situación económica de cada uno de ellos y los avances realizados al hilo de la aplicación de las recomendaciones adoptadas el año anterior. Informes que, en el caso de los Estados miembros con riesgo de desequilibrios macroeconómicos elevados, incluyen los resultados del examen exhaustivo llevado a cabo por la Comisión ${ }^{25}$.

Teniendo en cuenta estas orientaciones y los informes por país, en abril los Estados miembros presentan sus Programas Nacionales de Reformas (en adealnte, PNR), en los que informan a la Comisión de las políticas que están aplicando y de las reformas que pretenden llevar a cabo para cum-

Para más información vid., asimismo, COMISIÓN EUROPEA, «The autum package explained», disponible en www.ec.eurpa.eu (consultado el 23 de mayo de 2018).

22 Vid. Consejo Europeo/Consejo de la Unión Europea, «Cómo funciona el Semestre Europeo», op. cit.

${ }^{23}$ Ibid.

24 Entonces, el Consejo ECOFIN procede a adoptar la recomendación en la que establece dichas orientaciones generales tal y como prevé el Tratado. Vid. A. DASHWOOD, «Decision Making at the Summit», The Cambridge yearbook of European legal studies, vol. 3 (2000), pp. 79-105, en concreto p. 101.

${ }^{25}$ Vid. Consejo Europeo/Consejo de la Unión Europea, «Cómo funciona el Semestre Europeo», op. cit. 
plir con las orientaciones dadas desde la Unión, y sus Programas de Estabilidad (en adelante, PE) o Convergencia (en adelante, PC) (antes del 30 de abril), en los que se recogen los Objetivos Presupuestarios a Medio Plazo (en adelante, OPMP) que los Estados miembros han de alcanzar para garantizar la sostenibilidad de las finanzas públicas o un rápido avance hacia dicha sostenibilidad ${ }^{26}$. Comisión y Consejo examinan los OPMP y estos programas, y vigilan su aplicación ${ }^{27}$. El Consejo puede enviar recomendaciones para que los Estados miembros ajusten los mismos o adoptar una decisión por la que se constate que no se ha tomado ninguna medida efectiva ${ }^{28}$. También puede enviarse a los Estados recomendaciones preventivas para corregir los desequilibrios macroeconómicos ${ }^{29}$. Además, se establecen sanciones para el caso de que los Estados no sigan las recomendaciones: unas para la supervisión presupuestaria en la zona euro ${ }^{30} \mathrm{y}$ otras para la corrección efectiva de los desequilibrios macroeconómicos en dicha zona ${ }^{31}$.

Las recomendaciones del Consejo se adoptan en junio sobre la base de las recomendaciones específicas por país que presenta la Comisión en mayo. Por último, los jefes de Estado y de gobierno las refrendan y en julio el Consejo adopta las recomendaciones definitivas ${ }^{32}$, cumpliendo así con lo dispuesto en el art. 121.2 TFUE, que contempla el procedimiento para la adopción de las orientaciones generales en materia de política económica por el Consejo Europeo y de la recomendación en la que se fijan las mismas por parte del Consejo ${ }^{33}$.

A continuación, durante el segundo semestre del año (de julio a diciembre), el llamado Semestre Nacional, los Estados miembros ajustan sus pro-

26 Vid. arts. 2 bis, 4 y 8 de la versión consolidada del Reglamento (CE) núm. 1466/97.

27 Ibid., arts. 5, 6, 9 y 10.

${ }^{28}$ Ibid., arts. 10.2 y 6.2 .

29 Vid. art. 6 del Reglamento (UE) núm. 1176/2011.

30 Diferenciándose entre las sanciones a aplicar en la vertiente preventiva y la correctiva del PEC, siendo encargada la Comisión de recomendar al Consejo la adopción de la decisión imponiendo la sanción. Vid. Reglamento (UE) núm. 1173/2011 del Parlamento Europeo y del Consejo, de 16 de noviembre de 2011, sobre la ejecución efectiva de la supervisión presupuestaria en la zona del euro (DO, núm. L 306, de 23 de noviembre de 2011).

${ }^{31}$ Reglamento (UE) núm. 1174/2011 del Parlamento Europeo y del Consejo, de 16 de noviembre de 2011, relativa a las medidas de ejecución destinadas a corregir los desequilibrios macroeconómicos excesivos en la zona euro (DO, núm. L 306, de 23 de noviembre de 2011).

32 Vid. Consejo Europeo/Consejo de la Unión Europea, «Cómo funciona el Semestre Europeo», op. cit.

33 Como se ha señalado, la recomendación se adopta en julio, pero, como puede observarse, el proceso comienza con antelación. 
yectos presupuestarios nacionales a las orientaciones y recomendaciones recibidas, y la Unión Europea supervisa que las mismas son aplicadas ${ }^{34}$. A este respecto, las nuevas normas refuerzan la supervisión de los Estados miembros de la zona euro, debiendo los mismos presentar sus proyectos de planes presupuestarios para el año siguiente a más tardar el 15 de octubre, proyecto que ha de ser coherente con las recomendaciones formuladas en el contexto del PEC y del Semestre Europeo ${ }^{35}$. La Comisión examina los proyectos de planes presupuestarios y adopta un dictamen lo antes posible, a más tardar el 30 de noviembre, y puede pedir al Estado que revise el proyecto si constata un incumplimiento especialmente grave de las obligaciones de política presupuestaria establecidas en el PEC ${ }^{36}$. Tras ello, los Estados miembros adoptan sus proyectos de planes presupuestarios a finales de año. De esta forma, como señala Chalmers, se devalúan los procedimientos nacionales y la Comisión suplanta el papel de los Parlamentos, pues es en la Comisión donde tiene lugar el control de los presupuestos en primer lugar. Así, señala, los Parlamentos nacionales se debilitan y se refuerzan los ejecutivos, pues presentan unos proyectos de presupuestos que ya cuentan con el respaldo de la Comisión, dejando a los Parlamentos nacionales con poca opción más que ratificar el proyecto presentado a las instituciones de la Unión Europea ${ }^{37}$.

La vigilancia también se refuerza respecto de los Estados miembros que experimentan, o corren el riesgo de experimentar, graves dificultades desde el punto de vista de su estabilidad financiera o de la sostenibilidad de sus finanzas públicas que puedan tener efectos adversos en otros Estados miembros de la zona euro, y respecto de aquellos que soliciten o hayan recibido asistencia financiera ${ }^{38}$. Evidentemente, además de tener que seguir las recomendaciones que les hayan sido dirigidas en el

${ }^{34}$ Un esquema completo del Semestre Europeo puede consultarse también en la web de la Comisión Europea: http://ec.europa.euleconomy_financeleconomic_governancel the_european_semester/index_en.htm

${ }_{35}$ Vid. art. 6 del Reglamento (UE) núm. 473/2013 del Parlamento Europeo y del Consejo, de 21 de mayo de 2013, sobre disposiciones comunes para el seguimiento y la evaluación de los proyectos de planes presupuestarios y para la corrección del déficit excesivo de los Estados miembros de la zona euro (DO, núm. L 140, de 27 de mayo de 2013).

36 Ibid., art. 7.

37 D. Chalmers, «The European Redistributive State and a European Law of Struggle», European Law Journal, vol. 18, núm. 5 (2012), pp. 667-693, en concreto p. 687.

38 Vid. Reglamento (UE) núm. 472/2013 del Parlamento Europeo y del Consejo, de 21 de mayo de 2013, sobre el reforzamiento de la supervisión económica y presupuestaria de los Estados miembros de la zona euro cuya estabilidad financiera experimenta o corre el riesgo de experimentar graves dificultades. 
marco de la supervisión de su situación presupuestaria y de la supervisión y coordinación de sus políticas económicas, en el marco de un procedimiento de déficit excesivo, o por lo que respecta a los programas nacionales de reforma y programas de estabilidad, deberán adoptar medidas para atajar las causas reales o potenciales de las dificultades tras consultar y en cooperación con la Comisión, que actuará en coordinación con el Banco Central Europeo (en adelante, BCE), las diversas Autoridades Europeas de Supervisión (en adelante, AES), la Junta Europea de Riesgo Sistémico (en adelante, JERS) y, cuando proceda, el Fondo Monetario Internacional (en adelante, FMI) ${ }^{39}$. Y, por lo que respecta al seguimiento más estrecho de la situación presupuestaria, se les aplicarán también las previsiones señaladas para el seguimiento y evaluación de los planes presupuestarios nacionales ${ }^{40}$.

\section{LA SUPERVISIÓN DE ESPAÑA DENTRO DEL NUEVO SEMESTRE EUROPEO DE COORDINACIÓN ECONÓMICA}

El Semestre Europeo se puso en marcha en 2011 con el primer EPAC de la Comisión, comenzando así el nuevo ciclo de coordinación económica de la Unión Europea que se centraba en las medidas a adoptar para hacer frente a la crisis económica, comenzar la recuperación, corregir los desequilibrios macroeconómicos y volver a crear empleo.

Desde entonces se han llevado a cabo ocho ciclos de coordinación económica y actualmente nos encontramos en el nuevo ciclo para 2019, habiéndose presentado ya los PNR y los PE o PC, y presentado la Comisión sus propuestas de recomendación por países ${ }^{41}$. Examinaremos en el presente apartado las principales áreas en las que España ha debido llevar a cabo reformas para cumplir con las recomendaciones fijadas por el Consejo en el marco del Semestre Europeo, si bien destacaremos de las mismas solo algunas de las reformas adoptadas para obtener una visión general, pues el estudio exhaustivo de todas las reformas emprendidas desborda el

39 Ibid., art. 3.1.

40 Ibid., art. 3.2.

${ }^{41}$ Pueden consultarse a través de la web de la Comisión Europea los diversos documentos tanto del Semestre actualmente en curso como los de años anteriores: https://ec.europa.eu/info/business-economy-euro/economic-and-fiscal-policy-coordination/ eu-economic-governance-monitoring-prevention-correction/european-semester/european-semestertimeline_en. 
contenido del presente trabajo ${ }^{42}$. El objetivo es comprobar cómo las políticas económicas y presupuestarias del Estado español se han ajustado a las orientaciones y prioridades marcadas desde la Unión Europea.

\section{Semestre Europeo 2011}

Como se ha adelantado, el primer Semestre Europeo se puso en marcha en 2011, con el primer EPAC de la Comisión, el cual se centraba en un enfoque integrado basado en tres grandes ámbitos cubiertos por la Estrategia Europa 2020 $0^{43}$ :

- Un saneamiento fiscal riguroso para aumentar la estabilidad macroeconómica, de cara a conseguir un gasto público sostenible, manteniendo controlado el mismo, pero a la vez dando prioridad a un gasto que propiciase el crecimiento sostenible en sectores como la investigación, la educación y la energía; adoptando medidas para eliminar los déficits excesivos e incluso previendo la necesidad para algunos Estados de aumentar impuestos (preferiblemente los indirectos).

- Reformas en el mercado laboral para incrementar la tasa de empleo, por ejemplo, mediante medidas para lograr un equilibrio entre seguridad y flexibilidad en el trabajo (reformando la legislación en materia de despido, reduciendo la sobreprotección de los trabajadores con contratos indefinidos y brindando protección a quienes se encuentran fuera del mercado laboral).

- Medidas de fomento del crecimiento, mediante el aprovechamiento del potencial del mercado único o a través de la atracción de capital privado para financiar el mismo.

\footnotetext{
42 Señalar que se centra el análisis en los ciclos completados hasta el momento de entrega del presente artículo (el Semestre Europeo de 2018). Para el Semestre de 2019, en el momento de la última revisión del presente artículo ya se ha presentado el PNR y el PE, sobre la base de las recomendaciones específicas a España y el Informe País para 2019 que señalan la necesidad de garantizar la sostenibilidad fiscal, la realización de mejoras en la política social, educativa y de inserción laboral, y el aumento y mejora de la I+D+i, y el avance en la aplicación de la Ley General de Unidad de Mercado. Pueden consultarse a través de este enlace bttps://ec.europa.eu/info/business-economy-euro/economic-and-fiscal-policy-coordination/eueconomic-governance-monitoring-prevention-correction/european-semester/european-semesteryour-country/spain/european-semester-documents-spain_en.

${ }^{43}$ Vid. Primer Estudio Prospectivo Anual de la Comisión Europea 2011, COM (2011) 11 final.
} 
Estas prioridades fueron refrendadas tanto por el Consejo ${ }^{44}$ como por el Consejo Europeo de marzo del citado año ${ }^{45}$, y sobre la base de las prioridades y orientaciones dadas, el Estado español presentó su PNR y su PE. Mediante el PNR se fijaron los OPMP y las medidas de política económica para alcanzarlos. Así, dentro de la agenda reformadora del Gobierno para 2011 se incluían los tres grandes ámbitos señalados: un programa de consolidación fiscal para la reducción del déficit, el desarrollo de la reforma laboral que ya se había aprobado el año anterior de cara a su flexibilización, la reforma del sistema de pensiones y la reforma de la negociación colectiva, y las medidas para la recapitalización y reestructuración del sector financiero, entre otras ${ }^{46}$.

Por su parte, el PE preveía la reducción del déficit presupuestario para 2013 y 2014, si bien no se preveía alcanzar el objetivo de equilibrio presupuestario en el periodo abarcado por el programa ${ }^{47}$. Así, para cumplir con el objetivo de reducción del déficit el programa señalaba que las medidas de consolidación fiscal se basaban en ajustes del gasto no financieros, mejorando la eficiencia del gasto y reordenando y reestructurando el sector público, y presentando la propuesta de reforma del sistema público de pensiones de cara a garantizar la sostenibilidad de las finanzas públicas a largo plazo $^{48}$. Y recogía, asimismo, las reformas estructurales en otros ámbitos, como las adoptadas en relación con el mercado laboral (por ejemplo, en materia de contratación y despidos) o de cara a la reforma del sector financiero ${ }^{49}$.

Los citados programas fueron examinados por la Comisión ${ }^{50}$ y el Consejo, aprobando este último sus recomendaciones definitivas el 12 de julio de 2011, en las cuales recomendaba a España, entre otras cuestiones, la aplicación de la estrategia presupuestaria en 2011 y 2012 y la corrección del déficit excesivo en el año 2013; la adopción de nuevas medidas en caso

44 Vid. Sesión núm. 3067 del Consejo de la Unión Europea (Asuntos Económicos y Monetarios), celebrada en Bruselas el 15 de febrero de 2011, comunicado de prensa, documento 6514/11.

45 Vid. punto 2 de las Conclusiones del Consejo Europeo de 24 y 25 de marzo de 2011.

46 Vid. Programa Nacional de Reformas, p. 3. Puede consultarse a través de la web de la Comisión Europea ya indicada o desde la web del Gobierno de España en las referencias del Consejo de Ministros (www.moncloa.gob.es).

47 Vid. Programa de Estabilidad 2011-2014, pp. 4-5.

48 Ibid.

${ }^{49}$ Ibid., p. 6.

50 Tras ese examen, la Comisión aprueba las recomendaciones específicas por país, que son refrendadas por el Consejo Europeo en junio y posteriormente adoptadas por el Consejo en julio, por lo que se examinan brevemente estas últimas directamente. 
de que los resultados presupuestarios y económicos no se ajustasen a lo previsto, y la adopción de la reforma del sistema de pensiones propuesta con el fin de retrasar la edad de jubilación legal y aumentar el número de años de trabajo para el cálculo de las pensiones ${ }^{51}$.

\section{Semestre Europeo 2012}

Durante el segundo Semestre Europeo, la Comisión examinó brevemente los progresos realizados un año después de la puesta en marcha del primer Semestre, estableciendo como prioridades para 2012 la consolidación fiscal diferenciada favorable al desarrollo, el restablecimiento del crédito a la economía, la promoción del crecimiento y la competitividad presentes y futuros, la respuesta al desempleo y a las consecuencias sociales de la crisis, y la modernización de la Administración Pública. Así, se volvía a hacer referencia a la reforma y modernización de los sistemas de pensiones; a la continuación de la mejora de los sistemas fiscales y la posibilidad de que algunos Estados necesitasen volver a aumentar algunos impuestos, o a las reformas necesarias en el marco laboral (como la revisión de los mecanismos de fijación de salarios o la restricción del acceso a los regímenes de jubilación anticipada ${ }^{52}$. Seguían, por tanto, detectándose los tres principales ámbitos ya señalados en el primer EPAC de la Comisión: el saneamiento fiscal, la reforma del mercado laboral y las medidas de fomento del crecimiento.

De nuevo, el Consejo dio en febrero su acuerdo a las prioridades acordadas por la Comisión ${ }^{53}$ y la cumbre del Consejo Europeo de marzo las refrendó ${ }^{54}$. Prioridades que fueron tenidas en cuenta por el PNR presentado por España en 2012, en el que se señalaban, por ejemplo, las reformas puestas en marcha en la Administración Pública de cara a su sostenibilidad financiera, en el sistema sanitario en materia de Seguridad Social y, de

51 Vid. Recomendación del Consejo de 12 de julio de 2011 relativa al Programa Nacional de Reforma de 2011 de España y por la que se emite un dictamen del Consejo sobre el Programa de Estabilidad actualizado de España (2011-2014) (DOUE, núm. C 212, de 19 de julio de 2011).

52 Vid. Estudio Prospectivo Anual sobre el Crecimiento 2012, COM (2011) 815 final, Bruselas, 23 de noviembre de 2011, pp. 4-5 y 12.

53 Vid. Sesión núm. 3148 del Consejo de la Unión Europea (Asuntos Económicos y Monetarios), celebrada en Bruselas el 21 de febrero de 2012, comunicado de prensa, documento 6678/12, pp. 13 y ss.

54 Vid. punto 4 de las Conclusiones del Consejo Europeo de 1 y 2 de marzo de 2012. 
nuevo, las reformas en el mercado laboral para una mayor flexibilidad ${ }^{55}$. Y por lo que respecta a la actualización del PE para el periodo 2012-2015, se señalaba como objetivo de la política económica el saneamiento de las cuentas públicas y se fijaban los objetivos para la reducción del déficit presupuestario para 2013 y 2014, previendo alcanzar el equilibrio presupuestario en $2016^{56}$. Para cumplir estos objetivos el programa detallaba las medidas llevadas a cabo y las medidas a realizar, haciendo referencia, por ejemplo, a la reforma del sistema público de pensiones y al incremento progresivo de la edad de jubilación hasta los sesenta y siete años entre 2013 y $2027^{57}$. El programa abordaba también las medidas para la racionalización del gasto a través de la reestructuración de las Administraciones Públicas, la ampliación de bases imponibles y reducción de distorsiones en el sistema tributario, etcétera ${ }^{58}$.

Los programas fueron examinados por la Comisión y el Consejo, aprobando el Consejo sus recomendaciones definitivas el 10 de julio de 2012, en las que se recomendaba a España aplicar las reformas en el sector público para mejorar la eficiencia y la calidad del gasto público en todos los niveles de la Administración Pública, asegurar que la edad de jubilación fuese aumentando en función de la esperanza de vida, introducir un sistema tributario compatible con los esfuerzos de saneamiento presupuestario y más favorable al crecimiento, aplicar la reforma del sector financiero, aplicar las reformas del mercado laboral y tomar medidas adicionales encaminadas a aumentar la eficacia de las políticas activas del mercado de trabajo, etcétera ${ }^{59}$.

\section{Semestre Europeo 2013}

Siguiendo las recomendaciones mencionadas en los apartados anteriores, los Estados miembros fueron introduciendo reformas importantes para cumplir con las mismas y afrontar la crisis económica y financiera. Pero la recuperación seguía siendo lenta y difícil, especialmente en los

55 Vid. Programa Nacional de Reformas 2012.

56 Vid. Actualización del Programa de Estabilidad 2012-2015, p. 6.

57 Ibid., p. 74.

${ }^{58}$ Ibid.,pp. 71 y ss.

59 Vid. Recomendación del Consejo de 10 de julio de 2012 relativa al Programa Nacional de Reforma de 2012 de España y por la que se emite un dictamen del Consejo sobre el Programa de Estabilidad de España para 2012-2015 (DOUE, núm. C 219, de 27 de julio de 2012). 
países más afectados por la crisis. Así, en el inicio del Semestre Europeo de 2013 la Comisión reconocía que las reformas emprendidas ya iban surtiendo efecto, pero que había que seguir avanzando en las mismas, por lo que volvía a adoptar como prioridades para el nuevo año las ya indicadas en el anterior EPAC $^{60}$.

Las citadas prioridades fueron nuevamente refrendadas por el Consejo y por el Consejo Europeo en febrero y marzo de 2013, respectivamente ${ }^{61}$. Y de nuevo, el PNR de 2013 se correspondía con las prioridades acordadas a nivel de la Unión Europea, profundizando en las reformas iniciadas en 2012 encaminadas al aumento de la competitividad y, sobre todo, de la flexibilidad de la economía española (en materia de saneamiento de las cuentas públicas, reforma laboral, aumento de la competitividad de la economía española, reforma de las Administraciones Públicas, etc.), y exponiendo las medidas a poner en marcha en los siguientes meses ${ }^{62}$. En cuanto a la actualización del PE para 2013-2016, proyectaba en dos años más la corrección del déficit excesivo y señalaba las medidas adoptadas para el saneamiento fiscal de cara a la sostenibilidad de las finanzas públicas, para la reducción del déficit público, las iniciativas propuestas para asegurar el futuro del sistema de pensiones o las adoptadas para fomentar el crecimiento ${ }^{63}$.

Por último, tras el examen de los programas por la Comisión y por el Consejo, este presentó sus recomendaciones definitivas el 9 de julio de 2013, recomendando a España la corrección del déficit excesivo a más tardar en 2016, mejorar la eficiencia y la calidad del gasto público a todos los niveles de la Administración, realizar una revisión sistemática del sistema tributario para marzo de 2014, finalizar la evaluación de la reforma del mercado de trabajo de 2012 y presentar modificaciones, si fuese necesario, en septiembre de 2013 a más tardar, entre otras ${ }^{64}$.

60 Vid. Estudio Prospectivo Anual sobre el Crecimiento 2013, COM (2012) 750 final, p. 3.

${ }^{61}$ Vid. punto 1 del Documento de la Secretaría General del Consejo 5809/1/13 REV 1 y punto 1 de las Conclusiones del Consejo Europeo de 14 y 15 de marzo de 2013, respectivamente.

62 Vid. Programa Nacional de Reformas 2013, pp. 3 y 5-10.

63 Vid. Actualización del Programa de Estabilidad 2013-2016, pp. 1-5.

${ }^{64}$ Recomendación del Consejo de 9 de julio de 2013 relativa al Programa Nacional de Reformas de 2013 de España y por la que se emite un dictamen del Consejo sobre el Programa de Estabilidad de España para 2012-2016 (DOUE, núm. C 217, de 30 de julio de 2013). 


\section{Semestre Europeo 2014}

El cuarto Semestre Europeo comienza con la constatación de los primeros signos de recuperación en la Unión Europea y de la corrección de ciertos desequilibrios macroeconómicos importantes, siendo el mayor reto el «mantener el ritmo de las reformas para aumentar la competitividad y garantizar una recuperación duradera ${ }^{65}$. A dichos efectos, la Comisión volvía a proponer en su EPAC las cinco prioridades ya fijadas en 2012 y reiteradas en $2013^{66}$. Así, por ejemplo, de cara al saneamiento presupuestario volvía a establecer como prioridad la combinación de medidas de gasto e ingresos favorables al crecimiento, con una mayor atención a la calidad del gasto público, y en la modernización de la Administración a todos los niveles; para la lucha contra el desempleo y las consecuencias sociales de la crisis, reiteraba como prioridad las reformas en el mercado laboral, la modernización de los sistemas educativos y de formación, y la mejora del rendimiento de los sistemas de protección social, y en cuanto a la modernización de la Administración Pública, mantenía como prioridad la continuación de la implantación de los servicios de administración pública electrónica ${ }^{67}$.

Las prioridades fueron refrendadas por el Consejo Europeo de diciembre de 2013, que hacía hincapié en las políticas destinadas a aumentar la competitividad, apoyar la creación de empleo y combatir el desempleo, y en la actuación consecutiva a las reformas para mejorar el funcionamiento de los mercados laborales ${ }^{68}$.

De esta forma, el PNR de España para 2014 continuaba con la agenda reformista de los programas anteriores. La agenda económica del Gobierno contemplaba dos grandes líneas de actuación: profundizar en las reformas y favorecer la recuperación económica y la creación de empleo, estructurándose las medidas recogidas en el informe conforme a las cinco áreas prioritarias aprobadas desde la Unión Europea. Así, se recogían las medi-

65 Vid. Estudio Prospectivo Anual sobre el Crecimiento 2014, COM (2013) 800 final, p. 3.

${ }^{66}$ Ibid. p. 5.

${ }^{67}$ Ibid. pp. 9-16.

${ }^{68}$ Vid. puntos 23 y 25 de las Conclusiones del Consejo Europeo de 19 y 20 de diciembre de 2013. El Consejo refrendaba las prioridades, siguiendo las indicaciones del Consejo Europeo de diciembre de 2013, el 18 de febrero de 2014. Vid. Sesión núm. 3294 del Consejo de la Unión Europea (Asuntos Económicos y Financieros), celebrada en Bruselas el 18 de febrero de 2014, comunicado de prensa, documento 6566/14. 
das llevadas a cabo de cara a la consolidación fiscal, la revisión del sistema tributario español, el saneamiento del sector financiero, la evaluación de la reforma laboral y las reformas a nivel de la Administración, entre otras ${ }^{69}$. También se exponían las principales medidas que se pondrían en marcha en 2014 en esas cinco áreas ${ }^{70}$. Y por lo que respecta a la actualización del PE para 2014-2017, preveía que la demanda interna y la externa contribuirían positivamente al crecimiento, que continuaría aumentando la capacidad de financiación de la economía española, que a partir de 2014 se crearía empleo neto y mantenía el compromiso con la consolidación fiscal, corrigiéndose el nivel de déficit público ${ }^{71}$.

Por último, tras el examen de los programas el Consejo presentó sus recomendaciones definitivas el 8 de julio de 2014, proponiendo reforzar la estrategia presupuestaria a partir de 2014 con el objetivo de corregir el déficit excesivo de manera sostenible en 2016, a más tardar; impulsar nuevas medidas para reducir la segmentación del mercado de trabajo en aras de la calidad y sostenibilidad del empleo; aplicar en todos los niveles de la Administración las recomendaciones de la Comisión para la reforma de las Administraciones Públicas, etcétera ${ }^{72}$.

\section{Semestre Europeo 2015}

En el Semestre Europeo de 2015, el EPAC de la Comisión urgía a revitalizar el crecimiento en toda la Unión Europea, anunciando un nuevo paquete de medidas en favor del empleo, el crecimiento y la inversión $^{73}$. El estudio indicaba las medidas adicionales necesarias a adoptar a escala de la Unión Europea para ayudar a los Estados miembros a restablecer unos niveles de crecimiento más elevados, y para ello planteaba un enfoque integrado para propiciar el crecimiento, combinando las políticas estructurales, presupuestarias y monetarias. Enfoque integrado que se articulaba en torno a tres grandes pilares: el impulso coordi-

69 Vid. Programa Nacional de Reformas para 2014, pp. 1-32.

70 Ibid. pp. 39 y ss.

${ }^{71}$ Vid. Actualización del Programa de Estabilidad para 2014-2017, pp. 6-8.

72 Vid. recomendaciones 1, 3 y 8 de la Recomendación del Consejo de 8 de julio de 2014 relativa al Programa Nacional de Reformas de 2014 de España y por la que se emite un dictamen del Consejo sobre el Programa de Estabilidad de España para 2014 (DOUE, núm. 247, de 29 de julio de 2014).

73 Vid. Estudio Prospectivo Anual sobre el Crecimiento 2015, COM (2014) 902 final, Bruselas, 28 de noviembre de 2014, p. 3. 
nado de la inversión ${ }^{74}$; el compromiso renovado de emprender reformas estructurales (continuando con las reformas en el mercado laboral) ${ }^{75}$, y la perseverancia en la responsabilidad presupuestaria (debiendo los Estados miembros asegurar aún el control a largo plazo de sus niveles de déficit y de deuda) ${ }^{76}$.

Las prioridades de la Comisión fueron refrendadas por el Consejo en las conclusiones aprobadas en febrero de $2015^{77}$, estableciendo orientaciones para cada uno de los ámbitos marcados por la Comisión. Prioridades, asimismo, refrendadas por el Consejo Europeo en su reunión de marzo del citado año, que invitaba a los Estados miembros a trasladarlas a sus próximos PNR y sus $\mathrm{PE}$ o $\mathrm{PC}^{78}$.

De esta forma, el PNR de 2015 recogía las medidas correspondientes a la última fase de la agenda reformadora que el Gobierno había planteado para los cuatro años de legislatura. Por un lado, se señalaban las reformas abordadas para cumplir con las recomendaciones dadas en el marco del Semestre Europeo, en particular las formuladas en 2014: las llevadas a cabo de cara a la consolidación fiscal (reducción del déficit de las Administraciones, revisión del sistema tributario, lucha contra el fraude fiscal...); las adoptadas para el saneamiento del sector financiero (por ejemplo, la continuación de la reforma del sector de las cajas de ahorro); las establecidas en el marco del mercado laboral (reducción de cotizaciones a la Seguridad Social, avance en la transformación del modelo de políticas activas de empleo...), etcétera $^{79}$. Por otro, se exponían las principales medidas a poner en marcha a lo largo de 2015 en las tres áreas prioritarias señaladas por el EPAC de $2015^{80}$.

En cuanto a la actualización del PE para 2015-2018, mantenía el compromiso con la consolidación fiscal, cumpliendo con las recomendaciones para poner fin al PDE abierto a España en 2019. A este respecto, se describían las medidas a adoptar para alcanzar los objetivos de déficit público en los distintos niveles de la Administración. Y también se analizaban la sostenibilidad a largo plazo de las finanzas públicas y su calidad ${ }^{81}$.

74 Ibid., p. 5.

75 Ibid., p. 10. En materia de reforma laboral, se vuelve a hacer referencia a reformas en las normas de protección de empleo, de las pensiones, de los sistemas de protección social, etcétera. Vid. ibid., pp. 13 y ss.

${ }^{76}$ Ibid., pp. 5 y $16-18$.

77 Vid. documento 6147/15.

78 Vid. punto 4 de las Conclusiones del Consejo Europeo de 19 y 20 de marzo de 2015.

79 Vid. Programa Nacional de Reformas para 2015, pp. 11-39.

${ }^{80}$ Ibid., pp. 45-87.

81 Vid. Actualización del Programa de Estabilidad para 2015-2018, p. 10. 
Ambos programas fueron examinados por la Comisión y el Consejo, el cual, en sus recomendaciones definitivas, aconsejaba a España garantizar una corrección duradera del déficit excesivo en 2016, completar la reforma del sector de las cajas de ahorros, promover la adecuación de los salarios a la productividad y suprimir los obstáculos al crecimiento de las empresas ${ }^{82}$.

\section{Semestre Europeo 2016}

En su EPAC para 2016, la Comisión confirmaba las prioridades establecidas el año anterior ${ }^{83}$. El Consejo refrendaba dichas prioridades en sus conclusiones de enero de 2016 y establecía orientaciones con respecto a las mismas ${ }^{84}$. Prioridades que, a su vez, eran confirmadas por el Consejo Europeo en su reunión de marzo de $2016^{85}$.

El PNR de 2016 se caracterizó por la existencia de un gobierno en funciones, que en el programa daba cuenta de las medidas adoptadas en el último año y de las que entendía que sería necesario adoptar para consolidar los avances conseguidos. Así, respecto de las recomendaciones específicas recibidas durante el Semestre de 2015 se destacaba, por lo que respecta al ajuste presupuestario, la reducción del déficit público (con medidas para reforzar la disciplina fiscal en las Comunidades Autónomas de cara al control del gasto y el ajuste de las mismas a la regla de gasto, en aplicación de los mecanismos previstos en la Ley Orgánica de Estabilidad Presupuestaria); el saneamiento del sector financiero con la reestructuración de las entidades financieras (siguiendo las nuevas normas de la unión bancaria); la evolución del mercado de trabajo y el aumento de contrataciones (destacando las reformas en materia de negociación colectiva), y las reformas para la mejora de la competencia y el funcionamiento de los mercados (como, por ejemplo, la ley de segunda oportunidad para facilitar la resolución de las situaciones de insolvencia y favorecer el emprendimiento) ${ }^{86}$.

82 Vid. Recomendación del Consejo de 14 de julio de 2015 relativa al Programa Nacional de Reformas de 2015 de España y por la que se emite un dictamen del Consejo sobre el Programa de Estabilidad de 2015 de España (DOUE, núm. C 272, de 18 de agosto de 2015).

83 Vid. Estudio Prospectivo Anual sobre el Crecimiento 2016, p. 4.

${ }^{84}$ Vid. sesión del Consejo ECOFIN núm. 3442, de 15 de enero de 2016, disponible en bttp://www.consilium.europa.eu/es/press/press-releases/2016/01/15/conclusions -annual-growth-survey/.

${ }^{85}$ Vid. punto 11 de las Conclusiones del Consejo Europeo de 17 y 18 de marzo de 2016.

${ }^{86}$ Programa Nacional de Reformas para 2016, pp. 11-25. 
$Y$ en cuanto a las futuras reformas, entre otras, se recogían, por un lado, las reformas estructurales a nivel nacional [centradas en la creación de empleo (continuación de las reformas en las cotizaciones sociales, por ejemplo), las medidas de reforma de las Administraciones Públicas para control del gasto o la continuidad en el ajuste del déficit mediante una política fiscal racionalizadora del gasto, de forma que España pudiese salir del PDE lo antes posible], y, por otro, las reformas estructurales a nivel de la Unión Europea (en especial, siguiendo con el impulso de la reforma de la UEM) ${ }^{87}$.

En cuanto a la actualización del PE para 2016-2019, se seguía manteniendo el compromiso de reducción del déficit de cara a la consolidación fiscal y el cumplimiento de la regla de gasto, y se continuaba con un escenario macroeconómico prudente ${ }^{88}$. Así, se describían las medidas a adoptar en los distintos niveles de las Administraciones Públicas para alcanzar los objetivos de déficit y se analizaban nuevamente la sostenibilidad a largo plazo de las finanzas públicas y su calidad ${ }^{89}$.

Sobre el examen de los citados programas, la Comisión propuso un proyecto de recomendaciones específicas por país en mayo que fue examinado por el Consejo, que finalmente adoptó formalmente las mismas en julio de 2016, tras ser refrendadas por el Consejo Europeo ${ }^{90}$, donde volvía a recomendar a España la adopción de medidas para corregir de forma duradera el déficit excesivo (aplicando en todos los niveles de la Administración los instrumentos contemplados en la Ley Marco Presupuestaria, mejorando los mecanismos de control de la contratación pública y la coordinación de las políticas de contratación en todos los niveles de la Administración); la adopción de medidas adicionales para mejorar la integración en el mercado laboral y para mejorar la pertinencia de la enseñanza superior en el mismo, y acelerar la aplicación a nivel autonómico de la Ley de Garantía de la Unidad de Mercado ${ }^{91}$.

Asimismo, el 12 de julio el Consejo adoptaba una decisión por la que se establecía que España no había tomado medidas eficaces para corregir el déficit excesivo, siguiendo la recomendación del Consejo de 21 de junio

${ }^{87}$ Ibid., pp. 33-49.

${ }^{88}$ Actualización del Programa de Estabilidad para 2016-2019, pp. 4 у 8.

${ }^{89}$ Ibid., pp. 21 y ss.

90 Punto 9 de las Conclusiones del Consejo Europeo de 28 de junio de 2016.

${ }^{91}$ Recomendación del Consejo de 12 de julio de 2016 relativa al Programa Nacional de Reformas de 2016 de España y por la que se emite un dictamen del Consejo sobre el Programa de Estabilidad de 2016 de España (DOUE, núm. C 299, de 18 de agosto de 2016). 
de $2013^{92}$, si bien finalmente no se adoptó ninguna sanción tras valorar las reformas estructurales que se habían venido realizando al respecto.

\section{Semestre Europeo 2017}

En el EPAC de 2017 las prioridades en las que se insta a los Estados miembros a continuar redoblando sus esfuerzos continúan siendo la inversión, las reformas estructurales (en el mercado laboral, sistemas fiscales y sistemas de pensiones) ${ }^{93}$ y las políticas presupuestarias responsables (para continuar con la reducción del déficit público) ${ }^{94}$. El Consejo ECOFIN adoptaba sus conclusiones con respecto a dichas prioridades en su reunión de enero de 2017, fijando las respectivas orientaciones ${ }^{95}$, y el Consejo Europeo refrendaba las mismas en marzo, invitando como siempre a los Estados miembros a plasmarlas en sus PNR y PE o PC ${ }^{96}$.

En el PNR de 2017 el Gobierno español daba cuenta del grado de cumplimiento de las recomendaciones recibidas en el marco del Semestre Europeo de 2016 [en materia de reforma de las Administraciones Públicas, reforma fiscal (IRPF, impuesto de sociedades...), medidas contra el fraude fiscal y medidas en el marco del mercado laboral (fomento de la contratación indefinida o de jóvenes) $]^{97}$, así como las medidas para cumplir con las recomendaciones específicas por país [medidas para la reducción del déficit (control del gasto de Administraciones Públicas, revisión normativa, contratación pública...), mercado laboral, etc. ${ }^{98}$. Asimismo, se recogían las medidas para continuar con el crecimiento y el fomento del empleo. En cuanto a la actualización del PE 2017-2020 continúa en un escenario macroeconómico prudente, que prevé la reducción del déficit por debajo del 3 por 100 en $2018^{99}$.

Tras el examen de dichos programas por la Comisión y la adopción de su proyecto de recomendación, las recomendaciones definitivas al respecto

92 Decisión del Consejo por la que se establece que España no ha tomado medidas eficaces para seguir la Recomendación de 21 de junio de 2013 del Consejo, Bruselas, 11 de julio de 2016.

${ }_{93}$ Vid. Estudio Prospectivo Anual sobre el Crecimiento 2017, pp. 6 y ss.

${ }^{94}$ Ibid., pp. 17 y ss.

95 Vid. Conclusiones del Consejo ECOFIN de 27 de enero de 2017.

96 Vid. Conclusiones del Consejo Europeo de 9 de marzo de 2017.

97 Vid. Programa Nacional de Reformas de España para 2017, pp. 7-15.

${ }^{98}$ Ibid., pp. 16 y ss.

99 Vid. Programa de Estabilidad 2017-2020, p. 6. 
se adoptaron por el Consejo en su reunión de 11 de julio de 2017, tras ser refrendadas por el Consejo Europeo en su cumbre de 23 de junio ${ }^{100}$, recomendando a España tomar medidas para el cumplimiento de la recomendación de reducir el déficit excesivo, para el refuerzo de los marcos presupuestarios y de contratación pública, la revisión global de los gastos para aumentar su eficiencia, medidas para reforzar la coordinación entre servicios autonómicos de empleo, promover la contratación por tiempo indefinido, etcétera ${ }^{101}$.

\section{El Semestre Europeo en 2018}

El EPAC de 2018 vuelve a fijar como prioridades: la inversión, las reformas estructurales y las políticas presupuestarias responsables ${ }^{102}$, adoptando el Consejo ECOFIN sus conclusiones sobre las mismas en enero y fijando las orientaciones macroeconómicas y presupuestarias para los Estados miembros ${ }^{103}$, refrendando el Consejo Europeo en marzo los ámbitos de actuación prioritarios señalados en el EPAC de la Comisión ${ }^{104}$.

El PNR de 2018 una vez más da cuenta del grado de cumplimiento con las recomendaciones formuladas por el Consejo en el marco del Semestre Europeo [medidas de cara a cumplir con los objetivos de estabilidad presupuestaria y deuda pública en el marco del PEC (ámbito tributario para incrementar los ingresos, nueva ley de contratación pública...); en materia de empleo y políticas activas; en I+D+i, y en la cooperación entre Administraciones Públicas para garantizar la unidad del mercado] ${ }^{105}$, recogiendo las prioridades de política económica del Gobierno español para continuar con la senda del crecimiento y del empleo. La actualización del PE 2018-2021 continúa una vez más con el escenario macroeconómico prudente, manteniendo el compromiso de reducción del déficit público para situarlo en un 2,2 por 100 del PIB en 2018 y poder dar así por finalizado el PDE abierto a España ${ }^{106}$.

100 Vid. Conclusiones del Consejo Europeo de 23 de junio de 2017.

101 Vid. Recomendación del Consejo de 11 de julio de 2017 relativa al Programa Nacional de Reformas de 2017 de España y por la que se emite un dictamen del Consejo sobre el Programa de Estabilidad de 2017 de España (DOUE, núm. C 261, de 9 de agosto de 2017).

102 Vid. Estudio Prospectivo Anual sobre el Crecimiento 2018.

103 Vid. Conclusiones del Consejo ECOFIN de 23 de enero de 2018.

104 Vid. Conclusiones del Consejo Europeo de 22 de marzo de 2018.

105 Vid. Programa Nacional de Reformas de España para 2018, pp. 17 y ss.

106 Vid. Programa de Estabilidad 2018-2021, p. 6. Finalmente, España ha salido del PDE en junio de 2019. 
Ambos programas fueron examinados por la Comisión, adoptándose las recomendaciones específicas por país para 2018 por el Consejo en su sesión de 13 de julio de 2018, una vez refrendadas por el Consejo Europeo, en las que una vez más se recomienda a España tomar medidas para la reducción del déficit excesivo, a utilizar los beneficios para acelerar la reducción de la ratio de deuda pública, medidas en materia de empleo que apoyen eficazmente a los demandantes de empleo y favorezcan la transición hacia contratos indefinidos, aumento de la inversión pública en $\mathrm{I}+\mathrm{D}+\mathrm{i}$, etcétera ${ }^{107}$.

\section{CONCLUSIONES}

Como se ha analizado en el presente trabajo, las reglas adoptadas para la nueva gobernanza económica de la Unión Europea implican una coordinación y vigilancia más estrecha de las políticas económicas, fiscales y presupuestarias de los Estados miembros para asegurar la sostenibilidad de las finanzas públicas o un rápido progreso hacia dicha sostenibilidad. Políticas que tienen una gran importancia para un Estado, pues, por ejemplo, a través de las políticas económicas y presupuestarias los Estados intervienen en la economía para alcanzar determinados objetivos como la estabilidad de los precios (a corto plazo) o el crecimiento y el desarrollo económico (a largo plazo) ${ }^{108}$. Las nuevas normas afectan a la soberanía de los Estados miembros en estas áreas, que han visto cómo sus márgenes de decisión se han reducido ${ }^{109}$.

Como se ha analizado, en el marco del Semestre Europeo los Estados miembros han de remitir sus PNR y PE o PC, que son examinados por el Consejo y la Comisión, que envían recomendaciones a los Estados miembros y establecen plazos para el cumplimiento de las mismas ${ }^{110}$.

107 Vid. Recomendación del Consejo de 13 de julio de 2018 relativa al Programa Nacional de Reformas de 2018 de España y por la que se emite un dictamen del Consejo sobre el Programa de Estabilidad de 2018 de España (DOUE, núm. C 320, de 10 de septiembre de 2017).

108 Vid. J. Fernández Arufe (coord.), Principios de política económica, Madrid, Delta Publicaciones, 2006, pp. 21 y 26.

109 Especialmente la de aquellos Estados miembros que están experimentando serias dificultades en su estabilidad financiera y la sostenibilidad de sus finanzas públicas, así como los que han solicitado asistencia financiera, que son objeto de una vigilancia reforzada.

110 Es más, para los Estados miembros de la zona euro la vigilancia de sus políticas presupuestarias se refuerza, pasando sus proyectos presupuestarios a ser examinados antes de su adopción y pudiendo la Comisión exigir que se revisen si no se ajustan a las obligaciones de política presupuestaria establecidas en el PEC. Además, a través del TECG, los 
Las políticas económicas, fiscales y presupuestarias pasan, por tanto, a estar estrechamente vigiladas, con el objeto de controlar si se consigue reducir el déficit público, equilibrar los presupuestos nacionales y mantener unas finanzas públicas saneadas —o si se están dando los pasos adecuados para alcanzar dichos objetivos-.

Ese ha sido el caso de España, respecto del cual hemos analizado las principales áreas en las que se han abordado reformas para cumplir con las orientaciones y principios marcados desde la Unión Eurpea dentro del marco del Semestre Europeo. Así, ha debido realizar importantes reformas en sus políticas económicas, fiscales y presupuestarias, y adoptar toda una serie de medidas legislativas para cumplir con las exigencias de reducción del déficit y de saneamiento de las finanzas públicas. De esta forma, se han adoptado toda una serie de medidas de cara a la consolidación fiscal y la reducción del déficit público, controlando, por un lado, el gasto (habiéndose adoptado medidas para la reducción del gasto de las Administraciones Públicas) y, por otro, los ingresos (a través de las reformas en el sistema tributario). Y para garantizar la sostenibilidad de las finanzas públicas se han introducido reformas, por ejemplo, en el sistema público de pensiones y para el control del gasto sanitario. También se han realizado importantes reformas en el ámbito del mercado de trabajo, siguiendo nuevamente las recomendaciones adoptadas desde la Unión Europea para dotar al mismo de mayor flexibilidad (así, por ejemplo, se han introducido reformas en materia de contratación y despidos).

Los PNR y los PE presentados por España se han ajustado, como se ha examinado, a las prioridades y orientaciones marcadas desde el ámbito de la Unión, por lo que puede decirse que el ámbito de decisión del Estado español en estas áreas se ha visto también reducido.

El principal problema es la forma en que se han adoptado estas nuevas normas de gobernanza económica. Como se ha señalado, ha sido el Consejo Europeo quien ha estado en el origen de todas ellas, dando orientaciones precisas a las instituciones europeas e instándolas a adoptar las normas necesarias, imponiéndoles incluso plazos ${ }^{111}$. Además, ha creado nuevos

Estados miembros han acordado aplicar reglas más estrictas para promover la disciplina presupuestaria, reforzar la coordinación de sus políticas económicas y mejorar la gobernanza de la zona euro, que incluyen la incorporación en sus ordenamientos nacionales del principio de equilibrio presupuestario, preferentemente a través de normas de rango constitucional.

111 Utiliza, al efecto, un método de adopción de decisiones que lleva empleando desde hace tiempo: la adopción de la decisión a nivel político en sus reuniones y la activación posterior de los mecanismos de los Tratados para convertir la misma en normas jurídicas. 
métodos de trabajo, como el grupo especial que ha adoptado las recomendaciones que han dado origen al six pack, mecanismo a través del cual se codifica el Semestre Europeo. Institución que no está sujeta a ningún control político ni jurídico ${ }^{112}$, pues aunque el art. 10.2 TUE hace descansar el control en los Parlamentos nacionales, estos no disponen en la práctica de mecanismos para exigir responsabilidad individualmente a sus ministros, pues la institución requiere un control colegiado ${ }^{113}$.

Debe señalarse, asimismo, que el mecanismo utilizado para la adopción de las orientaciones generales de política económica y los nuevos métodos de trabajo introducidos por el Consejo Europeo - el grupo especialpresentan serios problemas de legitimidad democrática, son opacos y no permiten la participación de la opinión pública ${ }^{114}$. Y no puede olvidarse que a través de ellos el Consejo Europeo está dictando las reformas que los Estados miembros deben llevar a cabo, especialmente los que desean permanecer en la zona euro.

Además, tanto el TFUE como los nuevos reglamentos establecen que es el Consejo la institución que adopta las decisiones, contemplando como instrumento principal las recomendaciones e informando simplemente al Parlamento Europeo ${ }^{115}$. Recomendaciones que quedan excluidas del control del Tribunal de Justicia ${ }^{116}$. Medidas, por tanto, que siguen siendo intergubernamentales en lo que respecta a la adopción de decisiones ${ }^{117}$.

Se ha profundizado en un modelo de integración asimétrica y restrictiva que, como señala Rodríguez Ortiz, «conlleva renunciar a gran

Para más información sobre el proceso de decisión del Consejo Europeo vid. C. FERRER Martín de Vidales, La naturaleza del sistema institucional de la Unión Europea. Conclusiones del estudio del Consejo de la Unión, del Consejo Europeo y de la participación de los Parlamentos nacionales en la Unión Europea, tesis doctoral, Madrid, UCM, 2009, capítulo III, punto IV.

112 Vid. J. M. MarTínez SieRRA, El sistema institucional de la Unión Europea: la problemática presente y futura, tesis doctoral, Madrid, UCM, 2000, capítulo II.

113 Vid. J. M. Martínez Sierra y J. M. Díaz Martín, «Del control y la responsabilidad en la Unión Europea», Revista Universitaria Europea, núm. 3 (2002), pp. 132-164, en concreto p. 136, y Ph. KIIVER, The National Parliaments in the European Union: A Critical View on EU Constitution-Building, The Hague, Kluwer Law International, 2006, p. 73.

114 Vid. E. FERnÁNDEZ-MaCíAS, «La política laboral española en el seno de la UE. ¿Una integración sin competencias?, Revista Sistema, núm. 225-226 (2012), pp. 49-64, en concreto p. 58 .

115 Vid. arts. 120 a 126 TFUE y los reglamentos analizados en el segundo apartado del presente trabajo.

116 Vid. art. 263 TFUE.

117 Vid. J. I. Torreblanca y J. M. Areilza, «¿Cómo salir de la crisis del euro? Una visión desde España», Blogs ElPaís.com, 14 de junio de 2012, p. 1, disponible en http://blogs.elpais. $\mathrm{com} /$ files/torreblanca-areilza-2012-junio-salir-de-la-crisis-del-euro--1.pdf. 
parte de la autonomía del Estado en materia de política macroeconómica, lo que queda evidenciado con la constitucionalización de las reglas de equilibrio presupuestario (la mal llamada "regla de oro"), y esa renuncia supone sacrificar los pilares esenciales de la democracia política y social en Europa» ${ }^{118}$.

118 Vid. F. Rodríguez OrTIZ, «Crisis de la deuda y nueva gobernanza económica: una alternativa conservadora al gobierno económico europeo», comunicación al V Premio José Luis Sampedro, p. 22, disponible en http://xivrem.ujaen.es/wp-content/uploads/2011/11/2R-002M901.pdf. 